\title{
Prolonged Motor Weakness With Syringomyelia in Japanese Encephalitis: A Case Study
}

\author{
Young Moon Kim, MD, Youngkook Kim, MD, Jeehae Oh, MD, Hae Rim Kim, MD, Joo Hyun Park, MD, PhD
}

Department of Rehabilitation Medicine, Seoul St. Mary's Hospital,

College of Medicine, The Catholic University of Korea, Seoul, Korea

Japanese encephalitis (JE) shows characteristic brain lesions, including bilateral thalamus, midbrain, internal capsule, basal ganglia, and occasionally involves an anterior horn cell. We encountered a case of a 44-year-old man who initially presented with encephalitis, which was finally diagnosed as Japanese encephalomyelitis with syringomyelia. The patient showed severe motor weakness followed by delayed recovery of functional motor activities. Cervical magnetic resonance imaging showed syrinx formation at the C5 level suggesting myelitis, and abnormal electromyographic findings were noted. Clinicians should consider the possibility that the spinal cord may be involved; an example would be syringomyelia due to myelitis in a case of JE presenting with severe and prolonged motor weakness.

Keywords Japanese encephalitis, Myelitis, Syringomyelia

\section{INTRODUCTION}

Japanese encephalitis (JE) is one of the common causes of epidemic encephalitis in Asia. About $20 \%-40 \%$ of patients who develop encephalitis die, and around half of the survivors have severe neurological sequelae [1]. Brain magnetic resonance imaging (MRI) shows characteristic bilateral thalamic lesions involving the midbrain, internal capsule, and basal ganglia, which are associated with various clinical manifestations of movement disorders, such

Received June 10, 2014; Accepted October 16, 2014

Corresponding author: Joo Hyun Park

Department of Rehabilitation Medicine, Seoul St. Mary's Hospital, College of Medicine, The Catholic University of Korea, 222 Banpo-daero, Seocho-gu, Seoul 06591, Korea

Tel: +82-2-2258-6280, Fax: +82-2-2258-2825, E-mail: drpjh@catholic.ac.kr

(c) This is an open-access article distributed under the terms of the Creative Commons Attribution Non-Commercial License (http://creativecommons. org/licenses/by-nc/4.0) which permits unrestricted noncommercial use, distribution, and reproduction in any medium, provided the original work is properly cited.

Copyright $\odot 2015$ by Korean Academy of Rehabilitation Medicine as Parkinsonian syndromes. Anterior horn cell (AHC) damage manifesting as focal muscle wasting and lower motor neuron type of weakness has been documented in JE [2,3]. Verma et al. [4] reported a case of acute myelitis in JE, but syringomyelia as a result of myelitis has not been reported. We experienced a quadriplegic case with a prolonged clinical course of motor recovery as a result of not only encephalitis but also syrinx formation that was assumed to occur as a result of myelitis.

\section{CASE REPORT}

A 44-year-old male without any specific medical history visited the emergency department of our hospital with complaints of a headache and irritability that had been preceded by a fever for the past five days. After several hours, he had a generalized tonic seizure and respiratory insufficiency requiring endotracheal intubation. The cerebrospinal fluid analysis showed a white blood cell count of $45 / \mathrm{mm}^{3}$ (lymphocyte $92 \%$ ), protein level of 90.1 
$\mathrm{mg} / \mathrm{dL}$, and glucose level of $46 \mathrm{mg} / \mathrm{dL}$. On neurological examination, he showed a stuporous mental status and flaccid grade 3 paralysis in both upper limbs and grade 1 in both lower limbs. We used the Medical Research Council (MRC) grading. He was generally areflexic, and there was no pyramidal tract sign.

An initial brain MRI showed patchy and subtle T2 hyperintensity of both the hippocampal gyri and left thalamus. Follow-up imaging performed on the 28th day revealed more marked and extended lesions in the bilateral hippocampal gyri, thalamus, and basal ganglia (Fig. 1). At that time, nerve conduction studies (NCSs) showed slightly decreased amplitudes of bilateral median and ulnar compound motor action potentials (CMAPs) with normal distal latencies and conduction velocities. Furthermore, the somatosensory evoked potentials of median and tibial nerves revealed normal latencies. The diagnosis of JE was confirmed by positive JE virus IgM in enzyme-linked immunosorbent assay (ELISA) and rising titer in reverse transcription polymerase chain reaction on the 31st day and 40th day, respectively.

On the 62nd day, the patient was transferred to the rehabilitation unit. The motor power was grade 0 to 1 in the right upper and lower limbs, grade 2 in the left upper limb, and grade 3 in the left lower limb in terms of the MRC grading and profound muscle wasting was noted. The patient showed a mixed pattern of the deep tendon reflex (DTR) as follows: hyperactive knee jerk as opposed to hypoactive biceps, triceps and ankle jerk. His score of Modified Barthel Index (MBI) was zero.

On the 71st day, follow-up NCSs and electromyogra- phy (EMG) was performed using Medelec Synergy EMG equipment (Oxford Instrument Medical Ltd., Surrey, UK). It revealed decreased amplitudes of bilateral median CMAPs (Table 1) and profuse abnormal spontaneous activities (ASAs) in all sampled muscles of the upper and lower limbs, as well as the trunk (Table 2). The motor evoked potentials from both the abductor pollicis brevis and abductor hallucis muscles revealed decreased amplitudes of evoked potentials. A whole spine MRI on the 77th day showed syrinx formation at the C5 level of the spinal cord on the T2-weighted image (Fig. 2). A followup brain MRI and diffusion tensor tractography on the 86th day demonstrated an improvement in lesions of the hippocampal gyri, thalamus, and basal ganglia. In addition, the corticospinal tract revealed preserved integrity of the tract (Fig. 3). After physical and occupational therapy with sessions twice a day, five days a week, motor power was improved, progressing to grade 3 in the upper limbs and grade 3 to 4 in the lower limbs in terms of the MRC grading, and MBI also improved to 23 at discharge on the 104th day.

\section{DISCUSSION}

JE often involves the thalamus, brainstem, basal ganglia, hippocampus, and occasionally the AHCs of the spinal cord [3]. With involvement of the AHCs, focal muscle wasting and lower motor neuron type of weakness can be seen [3]. In this case, the patient presented with initial manifestation of flaccid paralysis and acute respiratory failure mimicking Guillain-Barre syndrome. Solomon
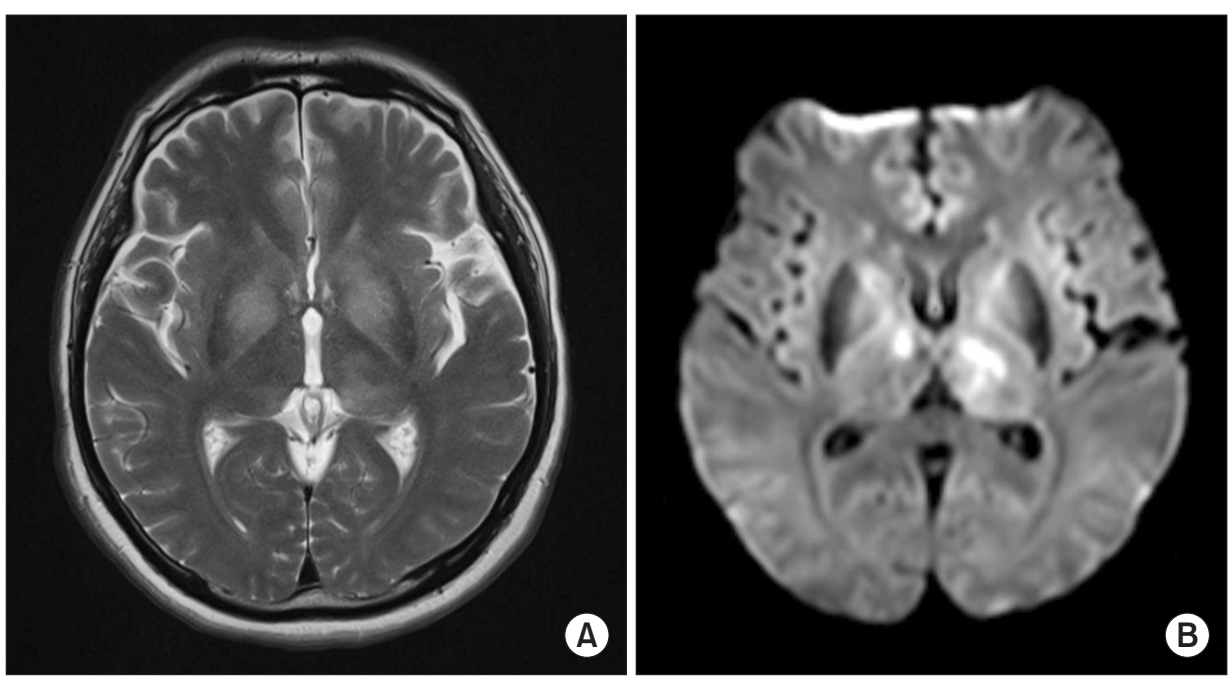

Fig. 1. Brain magnetic resonance imaging on the 28th day. (A) The axial T2-weighted image and (B) diffusion weighted image show characteristically high signal intensity in the bilateral thalamus and basal ganglia. 
Table 1. Findings of nerve conduction studies

\begin{tabular}{|c|c|c|c|c|c|}
\hline Nerve & Stimulation & Recording & Latency (ms) & Amplitude $^{\text {a) }}$ & $\mathrm{CV}(\mathrm{m} / \mathrm{s})$ \\
\hline \multicolumn{6}{|l|}{ Motor } \\
\hline \multirow{2}{*}{ Lt. Median } & Wrist & APB & 3.70 & 2.6 & - \\
\hline & Elbow & & 7.85 & 2.6 & 50.6 \\
\hline \multirow[t]{2}{*}{ Rt. Median } & Wrist & APB & 3.25 & 5.4 & - \\
\hline & Elbow & & 7.50 & 4.9 & 51.8 \\
\hline \multirow[t]{2}{*}{ Lt. Ulnar } & Wrist & $\mathrm{ADM}$ & 2.75 & 6.9 & - \\
\hline & B. Elbow & & 6.50 & 5.7 & 50.7 \\
\hline \multirow[t]{2}{*}{ Rt. Ulnar } & Wrist & $\mathrm{ADM}$ & 2.75 & 8.1 & - \\
\hline & B. Elbow & & 6.85 & 7.2 & 50.0 \\
\hline \multirow[t]{4}{*}{ Lt. Common peroneal } & Ankle & EDB & 4.75 & 2.1 & - \\
\hline & Fibular head & & 11.65 & 2.0 & 40.6 \\
\hline & Fibular head & TA & 4.25 & 3.9 & - \\
\hline & Knee & & 5.95 & 3.3 & 58.8 \\
\hline \multirow[t]{4}{*}{ Rt. Common peroneal } & Ankle & EDB & 5.00 & 0.9 & - \\
\hline & Fibular head & & 12.95 & 0.6 & 47.8 \\
\hline & Fibular head & TA & 3.80 & 3.0 & - \\
\hline & Knee & & 5.60 & 2.7 & 55.6 \\
\hline \multirow[t]{2}{*}{ Lt. Tibial } & Ankle & $\mathrm{AH}$ & 3.50 & 9.2 & - \\
\hline & Popliteal & & 11.60 & 8.3 & 49.4 \\
\hline \multirow[t]{2}{*}{ Lt. Tibial } & Ankle & $\mathrm{AH}$ & 3.65 & 7.5 & - \\
\hline & Popliteal & & 11.65 & 5.6 & 50.0 \\
\hline \multicolumn{6}{|l|}{ Sensory } \\
\hline Lt. Median & Wrist & Digit III & 3.35 & 30.7 & - \\
\hline Rt. Median & Wrist & Digit III & 3.25 & 36.7 & - \\
\hline Lt. Ulnar & Wrist & Digit V & 3.05 & 20.5 & - \\
\hline Rt. Ulnar & Wrist & Digit V & 3.25 & 20.8 & - \\
\hline Lt. Sural & Lateral mal & Calf & 3.75 & 10.9 & - \\
\hline Rt. Sural & Lateral mal & Calf & 3.15 & 11.9 & - \\
\hline Lt. Superficial peroneal & Ankle & Lateral leg & 3.30 & 8.4 & - \\
\hline Rt. Superficial peroneal & Ankle & Lateral leg & 3.15 & 11.9 & - \\
\hline
\end{tabular}

Lt., left; Rt., right; APB, abductor pollicis brevis; ADM, abductor digiti minimi; B elbow, below elbow; EDB, extensor digitorum brevis; TA, tibialis anterior; $\mathrm{AH}$, abductor hallucis; Lateral mal, lateral malleolus.

${ }^{a)}$ Amplitudes are measured in millivolts ( $\mathrm{mV}$, motor) and in microvolts $(\mu \mathrm{V}$, sensory).

et al. [2] reported flaccid paralysis in JE as an initial feature in Vietnamese children. In addition, there are some reports that have demonstrated prominent lower motor neuron type of weakness resembling that in GuillainBarre syndrome or bulbar poliomyelitis due to JE [3,5].

When transferred to the rehabilitative unit, he was still in a bed-ridden state with little improvement in motor weakness. Therefore, we suspected that he had other lesions in addition to brain lesions; this suspicion was based on the clues such as profound muscle atrophy and mixed pattern of the DTR that suggested both upper and lower motor neuron lesions. There were profuse ASAs in all four limbs and trunk muscles on the EMG. Those electrodiagnostic findings were comparable to a previous report by Misra and Kalita [3]. Therefore, MRI of the spine was performed, and it revealed syringomyelia at the C5 level, which might result from accompanying myelitis. Misra and Kalita [3] and Kumar et al. [6] reported 
Table 2. Findings of the needle electromyography

\begin{tabular}{|c|c|c|c|c|}
\hline \multirow{2}{*}{ Muscle } & \multirow{2}{*}{ PSW } & \multirow{2}{*}{ Fib } & \multirow{2}{*}{ Fasc } & \multirow{2}{*}{$\begin{array}{c}\text { MUAP } \\
\text { Recruitment }\end{array}$} \\
\hline & & & & \\
\hline B. Cervical paraspinalis (C4-T1) & $3+$ & $1+$ & - & \\
\hline B. Lumbar paraspinalis (L2-S1) & $3+$ & $1+$ & - & \\
\hline B. Gluteus maximus & $3+$ & $1+$ & - & Reduced \\
\hline B. Gluteus medius & $3+$ & $1+$ & - & Reduced \\
\hline B. Vastus medialis & $3+$ & $1+$ & - & Reduced \\
\hline B. Tibialis anterior & $3+$ & $1+$ & - & Reduced \\
\hline B. Peroneus longus & $3+$ & $2+$ & - & Reduced \\
\hline B. Gastrocnemius & $3+$ & $1+$ & - & Reduced \\
\hline B. Biceps brachii & $3+$ & $1+$ & - & Reduced \\
\hline B. Deltoid & $3+$ & $1+$ & - & Reduced \\
\hline B. Triceps & $3+$ & $1+$ & - & Reduced \\
\hline B. Flexor carpi radialis & $3+$ & $1+$ & - & Reduced \\
\hline B. Extensor digitorum communis & $3+$ & $1+$ & - & Reduced \\
\hline B. Abductor pollicis brevis & $3+$ & $1+$ & - & Reduced \\
\hline B. Abductor digiti minimi & $3+$ & $2+$ & - & Reduced \\
\hline
\end{tabular}

PSW, positive sharp wave; Fib, fibrillation; Fasc, fasciculation; MUAP, motor unit action potential; B., bilateral.
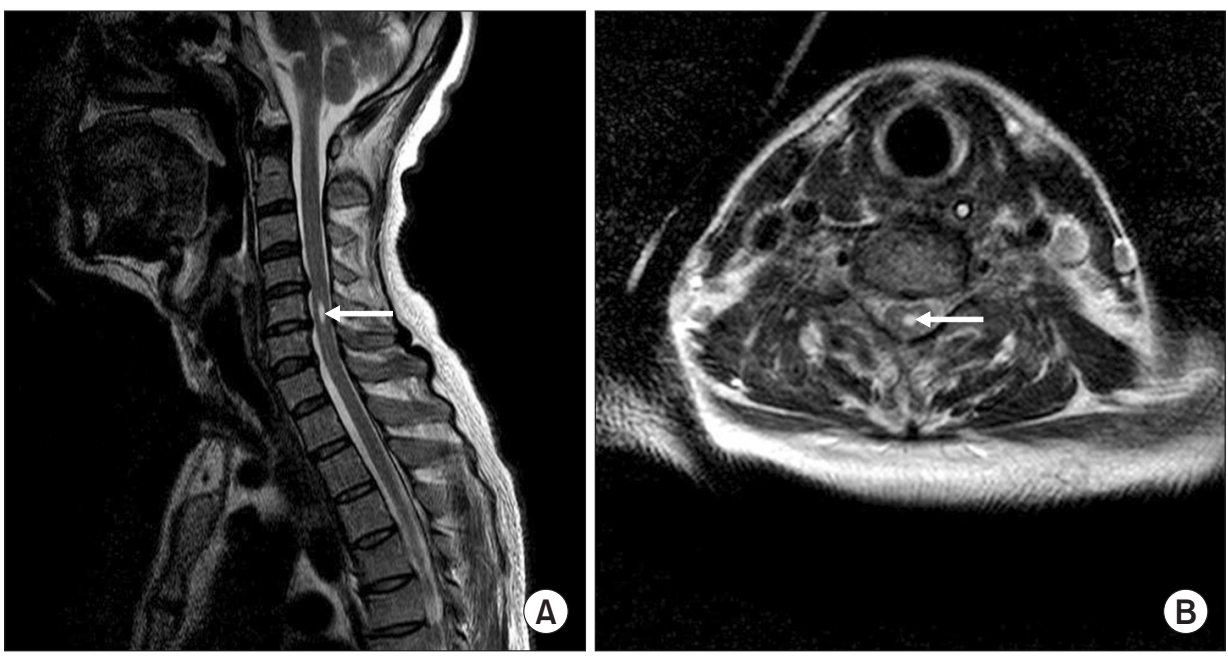

Fig. 2. Cervical magnetic resonance imaging on the 77th day. (A) Sagittal and (B) axial T2weighted images show a cystic lesion with high signal intensity, suggesting syrinx formation (arrows) at the C5 level.

increased signal changes on T2-weighted images of the spinal cord in JE patients. However, they did not report the specific location and the nature of the cord lesions.

Syringomyelia can result from a hindbrain anomaly (Chiari malformation), trauma, tumor, and arachnoiditis [7], but we can exclude these conditions by evaluating a patient's previous medical history and imaging studies. It has been reported that parainfectious myelitis can occur after several viral infections including JE possibly due to an immune-mediated mechanism [8], and inflam- matory cord lesions, such as myelitis, may contribute to syringomyelia formation [9]. Verma et al. [4] reported a case with acute transverse myelitis following JE which showed diffuse high signal intensity along a cervicothoracic spinal cord on T2-weighted image. Moreover, some authors have suggested that patients with spinal cord involvement were more likely to suffer from severe diseases with poor prognosis [2,3]. In addition, Solomon et al. [1] reported persistent paralysis one to two years later in JE patients. Our patient did not show significant motor re- 

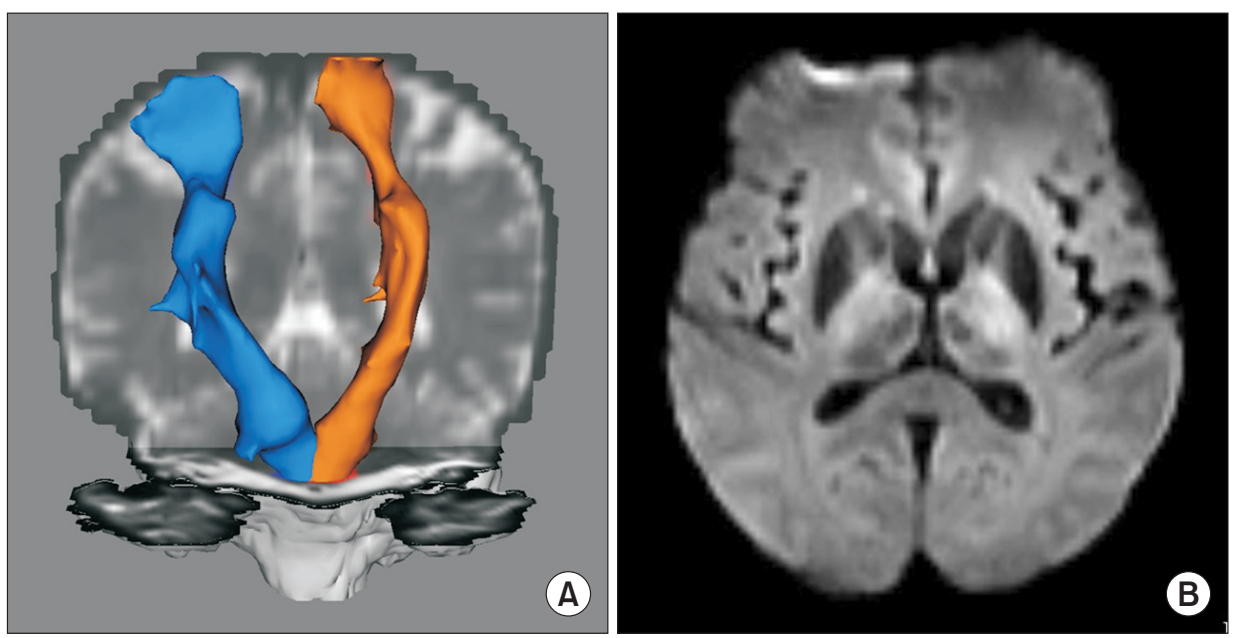

Fig. 3. Brain magnetic resonance imaging and diffusion tensor tractography (DTT) on the 87th day. (A) The DTT demonstrates the preserved integrity of both corticospinal tracts. (B) The diffusion weighted image shows the regression of high signal intensity in the bilateral thalamus and basal ganglia.

covery, and profound motor weakness persisted for two months after the onset. The formation of syringomyelia, which may affect prolonged motor weakness, was possibly subacute rather than acute due to time-consuming pathogenesis of sustained vasogenic edema and the direct inflammatory effect on the spinal cord, although the definite timing is not clear [9].

This case revealed that prolonged motor weakness might be attributed to syringomyelia complicated by preceding myelitis in JE. A spinal cord lesion in this case must be involved although a clinician might misunderstand JE as 'encephalitis' only-meaning brain damage. Therefore, a clinician should consider the possibility that the spinal cord may be involved in order to identify appropriate rehabilitative interventions when a patient with JE manifests unusual neurologic signs and a clinical course.

\section{CONFLICT OF INTEREST}

No potential conflict of interest relevant to this article was reported.

\section{REFERENCES}

1. Solomon T, Dung NM, Kneen R, Gainsborough M, Vaughn DW, Khanh VT. Japanese encephalitis. J Neurol Neurosurg Psychiatry 2000;68:405-15.
2. Solomon T, Kneen R, Dung NM, Khanh VC, Thuy TT, Ha DQ, et al. Poliomyelitis-like illness due to Japanese encephalitis virus. Lancet 1998;351:1094-7.

3. Misra UK, Kalita J. Anterior horn cells are also involved in Japanese encephalitis. Acta Neurol Scand 1997;96:114-7.

4. Verma R, Praharaj HN, Patil TB, Giri P. Acute transverse myelitis following Japanese encephalitis viral infection: an uncommon complication of a common disease. BMJ Case Rep 2012 Sep 24 [Epub]. http:// dx.doi.org/10.1136/bcr-2012-007094.

5. Tzeng SS. Respiratory paralysis as a presenting symptom in Japanese encephalitis: a case report. Zhonghua Yi Xue Za Zhi (Taipei) 1989;43:208-12.

6. Kumar S, Misra UK, Kalita J, Salwani V, Gupta RK, Gujral R. MRI in Japanese encephalitis. Neuroradiology 1997;39:180-4.

7. Fischbein NJ, Dillon WP, Cobbs C, Weinstein PR. The "presyrinx" state: a reversible myelopathic condition that may precede syringomyelia. AJNR Am J Neuroradiol 1999;20:7-20.

8. Goh C, Desmond PM, Phal PM. MRI in transverse myelitis. J Magn Reson Imaging 2014;40:1267-79.

9. Ravaglia S, Bogdanov EI, Pichiecchio A, Bergamaschi R, Moglia A, Mikhaylov IM. Pathogenetic role of myelitis for syringomyelia. Clin Neurol Neurosurg 2007;109:541-6. 